\title{
New onset diabetes mellitus and cardiovascular events in Korean patients with acute myocardial infarction receiving high-intensity statins
}

Jah Yeon Choi ${ }^{1}$, Cheol Ung Choi ${ }^{1 *}$ (D), Byoung Geol Choi ${ }^{1}$, Yoonjee Park', Dong Oh Kang ${ }^{1}$, Won Young Jang ${ }^{2}$,

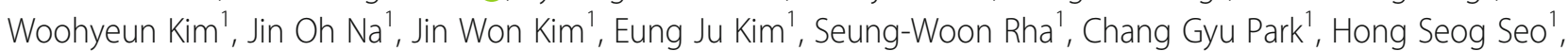
Myung Ho Jeong ${ }^{3}$, Sung-Chull Chae ${ }^{4}$, In-Whan Seong ${ }^{5}$, Chang-Hwan Yoon ${ }^{6}$, Kwang Soo Cha', Seok Kyu Oh ${ }^{8}$ and KAMIR-NIH Investigators

\begin{abstract}
Background: High-intensity statin therapy is typically used in patients with acute myocardial infarction (AMI) for secondary prevention. However, there have been consistent concerns regarding its association with diabetes mellitus. We investigated the effect of high-intensity atorvastatin and rosuvastatin on new-onset diabetes mellitus (NODM) and cardiovascular outcomes over a 3-year follow-up period.

Methods: Data from the Korea Acute Myocardial Infarction Registry were collected from November 2011 to October 2015, and 13,104 patients with AMI were enrolled from major cardiovascular centers. Among them, 2221 patients without diabetes who had been administered with high-intensity atorvastatin $(40-80 \mathrm{mg})$ and rosuvastatin $(20 \mathrm{mg})$ were investigated. The atorvastatin and rosuvastatin groups were evaluated for the incidence of NODM and major adverse cardiac events (MACE) including death, myocardial infarction, and revascularization cases in the following 3 years.
\end{abstract}

Results: Baseline characteristics were comparable between the two groups. Event-free survival rate of NODM was not significantly different between the atorvastatin and rosuvastatin groups ( $92.5 \%$ vs. 90.8\%, respectively; Log-rank $P$-value $=0.550$ ). The event-free survival rate of MACE was also not significantly different between atorvastatin and rosuvastatin groups ( $89.0 \%$ vs. $89.6 \%$, respectively; Log rank P-value $=0.662$ ). Multivariate Cox analysis revealed that statin type was not a prognostic factor in the development of NODM and MACE.

Conclusions: Administering high-intensity atorvastatin and rosuvastatin in patients with AMl produced comparable effects on NODM and clinical outcomes, suggesting their clinical equivalence in secondary prevention.

Keywords: Acute myocardial infarction, New-onset diabetes mellitus, Cardiovascular outcome, Atorvastatin, Rosuvastatin

\footnotetext{
* Correspondence: wmagpie@korea.com

${ }^{1}$ Cardiovascular Center, Korea University Guro Hospital, 80, Guro-dong, Guro-gu, Seoul 152-703, Republic of Korea

Full list of author information is available at the end of the article
} 


\section{Background}

Statins typically prevent cardiovascular events by lowering total and low-density lipoprotein (LDL) cholesterol levels in the serum. Considering their rapid and sustained clinical advantages, the current guideline recommends administration of high-intensity statins in patients with acute myocardial infarction (AMI) for secondary prevention. However, there has been consistent concern regarding its association with new-onset diabetes mellitus (NODM) [1]. Clinical trials, meta-analyses of randomized controlled trials (RCTs), and observational studies have demonstrated a $10-12 \%$ increase in NODM among patients receiving statins $[2,3]$. Meta-analysis of five large-scale trials comparing intensive and moderate doses of statins have demonstrated that the risk of NODM further increases in intensive therapy groups [4]. However, it is unclear if the diabetogenic effect of statins is a class effect. Considering their crucial role of secondary prevention in patients with AMI, it would be important to identify the diabetogenic and cardioprotective effects of high-intensity statins. In Korea, atorvastatin 40-80 mg and rosuvastatin $20 \mathrm{mg}$ are currently available as high-intensity statins for clinical use. Here, we investigated the effect of highintensity atorvastatin and rosuvastatin on cardiovascular outcomes and NODM in patients with AMI over a 3-year follow-up period.

\section{Methods}

\section{Data collection and study population}

The Korea Acute Myocardial Infarction Registry (KAMI $\mathrm{R})$, a Korean prospective, multicenter, nationwide database supported by the Korean Society of Cardiology, reflects real-world clinical practices of AMI patients in Asian. Twenty university or community hospitals have participated in the registry. Data collection at each institution level is performed by a study coordinator using a standardized case report form and the collected data are managed using web-based systems. The study was approved by the ethics committee at each institution. All the patients enrolled the study provided written informed consent.

A total of 13,104 patients with AMI were enrolled in the KAMIR registry from November 2011 to October 2015. Among them, 6728 patients without a diagnosis of diabetes mellitus (DM) at enrollment, with a successful percutaneous coronary intervention (PCI) with drugeluting stent implantation and high-intensity statin treatment were eligible for our study. Patients were selected considering the following exclusion criteria: history of $\mathrm{DM}$ or initial $\mathrm{HbA} 1 \mathrm{c}$ level $\geq 6.5 \%$, PCI with bare metal stent implantation or plain old balloon angioplasty. Additionally, we did not include patients with failed PCI, or in-hospital major adverse cardiac events (MACE). Finally, 2221 patients with AMI treated with high-intensity atorvastatin or rosuvastatin, according to 2014 ACC/AHA Release Updated Guideline, were analyzed in the study. Of them, $60.7 \%$ (1349/2221) of patients had received 40-80 $\mathrm{mg}$ atorvastatin and $39.3 \%(872 / 2221)$ had received $20 \mathrm{mg}$ rosuvastatin (Fig. 1).

\section{Clinical outcome and definition}

The primary endpoint was the incidence of NODM and the incidence of MACE during the 3 years of clinical follow-up. Secondary endpoints were each component of MACE, reasons for mortality, MI, and revascularization. NODM was defined as an HbA1c level $\geq 6.5 \%$ or new administration of oral hypoglycemic agents. The patients' clinical data were obtained by face-to-face interviews during regular outpatient visits, medical chart reviews, and telephonic interviews.

\section{Statistical analysis}

For continuous variables, differences between the two groups were analyzed by Student's t-test. Data were

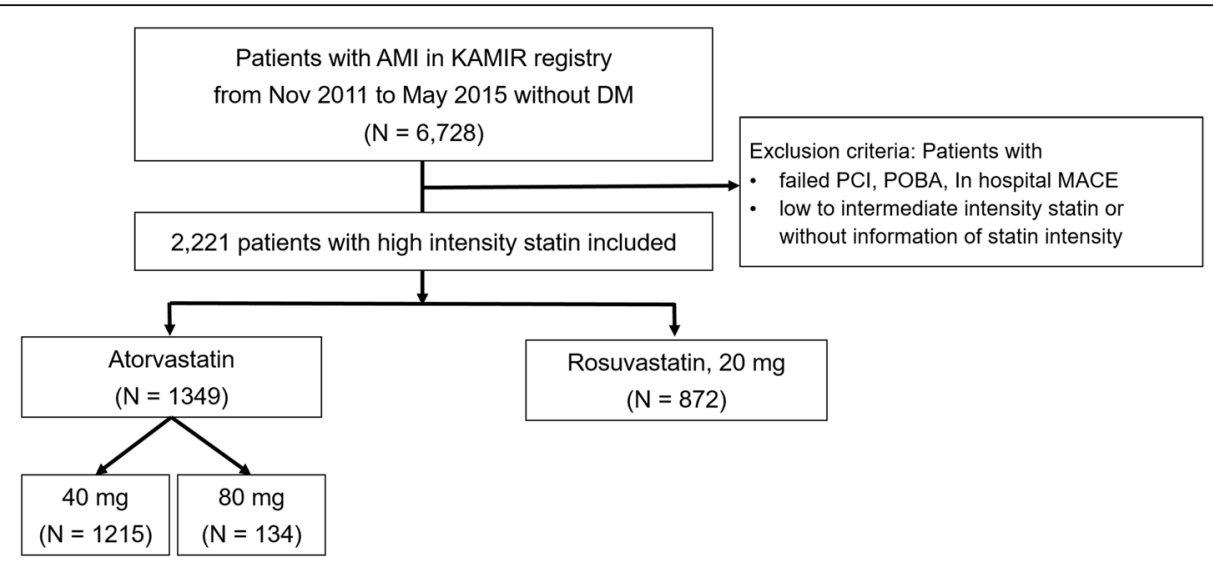

Fig. 1 Patient flow chart. AMI: Acute myocardial infarction, DM: diabetes mellitus, KAMIR: Korea Acute Myocardial Infarction Registry, MACE: major adverse cardiac events, $\mathrm{PCl}$ : percutaneous coronary intervention, POBA: plain old balloon angioplasty 
presented as mean \pm standard deviation (SD). For discrete variables, differences were analyzed using the Chi-square test or Fisher's exact test and presented as counts and percentages. The cumulative incidence of NODM and MACE was calculated using the Kaplan-Meier method, and the intergroup differences were analyzed using the log-rank test. Cox-proportional hazard models reporting hazard ratio (HR) and 95\% confidence interval (CI) was performed to identify potential prognostic factors for NODM and MACE. For multivariate analysis, variables with significant $P$-values $(<0.05)$ in the univariate analysis were included. For all analyses, a P-value $<0.05$ was considered statistically significant. The data were analyzed using SPSS (version 22.0, Inc. Chicago, IL).

\section{Results}

Baseline characteristics

Baseline clinical, laboratory, and angiographic characteristics are demonstrated in Table 1. We did not observe any significant intergroup differences with regard to age, gender, LV systolic function, incidence of STEMI, and underlying diseases such as hypertension and cerebrovascular accidents. Patients in the rosuvastatin group had higher levels of LDL cholesterol and peak CK-MB and a longer total stent length than those in the atorvastatin group. Dual antiplatelet therapy (DAPT) rate was above $99 \%$ in both groups; however, the composition of DAPT was different in that the prescription rate of prasugrel was higher in

Table 1 Baseline clinical characteristics and angiographic and procedural characteristics

\begin{tabular}{|c|c|c|c|}
\hline Variables & Atorvastatin $(n=1349)$ & Rosuvastatin $(n=872)$ & $P$-value \\
\hline Men & 1105 (81.9\%) & $720(82.6 \%)$ & 0.693 \\
\hline Age (years) & $61.0 \pm 12.5$ & $61.0 \pm 12.6$ & 0.918 \\
\hline LV ejection fraction (\%) & $54.1 \pm 9.9$ & $54.0 \pm 9.6$ & 0.906 \\
\hline Body mass index $\left(\mathrm{kg} / \mathrm{m}^{2}\right)$ & $24.5 \pm 3.2$ & $24.4 \pm 3.4$ & 0.442 \\
\hline \multicolumn{4}{|l|}{ Myocardial infarction } \\
\hline ST-segment elevation & 719 (53.3\%) & $478(54.8 \%)$ & 0.483 \\
\hline Non-ST-segment elevation & $630(46.7 \%)$ & $394(45.2 \%)$ & \\
\hline Hypertension & $549(40.7 \%)$ & 329 (37.7\%) & 0.162 \\
\hline Cerebrovascular accidents & $42(3.1 \%)$ & $29(3.3 \%)$ & 0.781 \\
\hline Total cholesterol (mg/dl) & $195.5 \pm 42.0$ & $196.6 \pm 45.6$ & 0.563 \\
\hline Triglyceride (mg/dl) & $146.5 \pm 120.3$ & $145.4 \pm 139.1$ & 0.852 \\
\hline HDL-cholesterol (mg/dl) & $44.1 \pm 11.1$ & $44.0 \pm 11.1$ & 0.790 \\
\hline LDL-cholesterol (mg/dl) & $126.5 \pm 36.3$ & $130.6 \pm 40.3$ & 0.020 \\
\hline CK-MB (mg/dl) & $125.2 \pm 159.2$ & $139.9 \pm 145.1$ & 0.029 \\
\hline Glucose (mg/dl) & $136.7 \pm 37.4$ & $138.8 \pm 37.9$ & 0.216 \\
\hline Creatinine (mg/dl) & $0.97 \pm 0.94$ & $0.94 \pm 0.42$ & 0.340 \\
\hline \multicolumn{4}{|l|}{ Discharge medications } \\
\hline Aspirin & 1343 (99.6\%) & 865 (99.2\%) & 0.280 \\
\hline Clopidogrel & $855(63.4 \%)$ & $473(54.2 \%)$ & $<0.001$ \\
\hline Prasugrel & $188(13.9 \%)$ & $68(7.8 \%)$ & $<0.001$ \\
\hline Ticagrelor & $302(22.4 \%)$ & $325(37.3 \%)$ & $<0.001$ \\
\hline Cilostazol & $57(4.2 \%)$ & $29(3.3 \%)$ & 0.283 \\
\hline Calcium channel blockers & $63(4.7 \%)$ & $45(5.2 \%)$ & 0.600 \\
\hline$\beta$ blockers & $1181(87.5 \%)$ & 714 (81.9\%) & $<0.001$ \\
\hline ACEi & $687(50.9 \%)$ & $321(36.8 \%)$ & $<0.001$ \\
\hline ARB & $426(31.6 \%)$ & 336 (38.5\%) & 0.001 \\
\hline \multicolumn{4}{|l|}{ Procedural characteristics } \\
\hline Total stent length (mm) & $27.6 \pm 12.2$ & $39.8 \pm 13.4$ & $<0.001$ \\
\hline Total stent number & $1.17 \pm 0.41$ & $1.20 \pm 0.45$ & 0.077 \\
\hline Stent diameter (mm) & $3.20 \pm 0.44$ & $3.14 \pm 0.44$ & 0.062 \\
\hline
\end{tabular}

$A C E i$ angiotensin-converting enzyme inhibitor, $A R B$ angiotensin receptor blocker, $C K-M B$ Creatine Kinase-MB, $H D L$ high-density lipoprotein, $L D L$ low-density lipoprotein, $L V$ left ventricle 
the atorvastatin group and that of ticagrelor was higher in the rosuvastatin group. Numbers of patients taking ACEi or ARB and $\beta$ blockers were higher in the atorvastatin group than in the rosuvastatin group.

\section{Clinical outcomes}

The cumulative incidence of NODM up to 3 years using Kaplan-Meier method is presented in Fig. $2 \mathrm{a}$ and Table 2. There was no significant difference in the event-free survival rate of NODM between the atorvastatin and rosuvastatin groups $(92.5 \%$ vs. $90.8 \%$, respectively; Logrank $P$-value $=0.550)$. Kaplan-Meier curves for the cumulative incidence of MACE up to a period of 3 years are presented in Fig. $2 \mathrm{~b}$ and Table 2. There was no significant difference between the atorvastatin and rosuvastatin groups regarding the event free survival rate of MACE $(89.0 \%$ vs. $89.6 \%$, respectively; Log rank P-value $=0.662)$, reasons for mortality, myocardial infarction, and revascularizations. Comparing $40 \mathrm{mg}$ and $80 \mathrm{mg}$ of atorvastatin groups with $20 \mathrm{mg}$ of rosuvastatin group revealed no significant differences in the event-free survival rate of NODM and MACE (see Additional file 1: Fig. S1A and B).

Potential prognostic factors for NODM were identified via univariate Cox regression analysis. Higher random glucose and triglyceride levels were both significant prognostic factors for NODM in univariate and multivariate analysis. However, the type of statin used was not ( $\mathrm{HR}=1.098,95 \%$ confidence interval [CI]: 0.808-1.491, $P=0.551$, Table 3). Conventional risk factors including older age, lower left ventricular ejection fraction (LVEF), and higher creatinine levels were associated with a higher incidence of MACE. Use of new antiplatelet agents such as ticagrelor or prasugrel was a significant prognostic factor in univariate analysis, not however, in the multivariate analysis. The type of high-intensity statin, atorvastatin or rosuvastatin, was not a potential prognostic factor for MACE $(\mathrm{HR}=0.944 \%$ confidence interval $[\mathrm{CI}]$ : $0.727-1.225, P=0.662$, Table 3 ).

\section{Discussion}

To the best of our knowledge, this is the first study to investigate the effect of high-intensity statin treatment on the development of NODM and MACE in Korean patients with AMI. Our results indicated that highintensity atorvastatin and rosuvastatin therapies showed no significant difference with regard to the incidence of NODM and cardiovascular events.

Statins reduce serum LDL cholesterol level and the risk of cardiovascular events. As numerous studies revealed that the degree of cardiovascular risk reduction is proportional to the statin intensity $[5,6]$, the current guidelines strongly recommend high-intensity or maximally tolerated intensity statin therapy in patients with AMI in the absence of contraindications [7, 8]. However, several studies have suggested that statins increase the incidence of NODM $[3,9]$. The issue has started attracting attention since the Justification for the Use of Statin in Prevention: An Intervention Trial Evaluating Rosuvastatin (JUPITER) trial revealed a higher incidence of NODM in rosuvastatin treated patients for primary prevention than in patients with placebo [10]. Numerous observational studies [11, 12] and meta-analyses of major RCT $[3,13]$ have consistently reported an increased incidence of NODM in patients treated with statin. In Korea, a population-based cohort study using the Korean National Health Insurance claims database has shown an increased incidence of NODM in statin-treated groups [2].

Whether the diabetogenic effect of statin is a class effect has been a controversial subject. Typically, atorvastatin and rosuvastatin are thought to unfavorably influence
A. New Onset Diabetes Mellitus

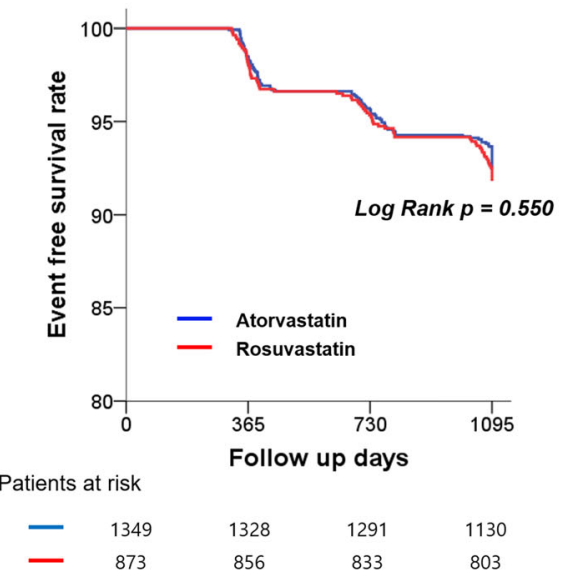

B. Major Adverse Cardiac Events

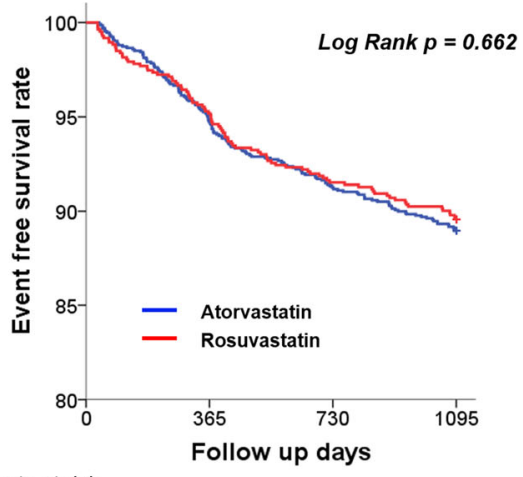

Patients at risk

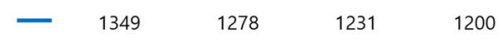

Fig. 2 Kaplan-Meier curves for event-free survival rate of new-onset diabetes mellitus (a) and major adverse cardiac events (b) according to statin type 
Table 2 Cardiovascular Outcomes at 3 Years

\begin{tabular}{llll}
\hline Clinical outcome & Atorvastatin $(\boldsymbol{n = 1 3 4 9 )}$ & Rosuvastatin $(\boldsymbol{n}=\mathbf{8 7 2})$ & $\boldsymbol{P}$-value \\
\hline New onset diabetes mellitus & $99(7.5)$ & $70(9.2)$ & 0.550 \\
MACE & $149(11.0)$ & $91(10.4)$ & 0.662 \\
All cause of mortality & $49(3.6)$ & $25(2.9)$ & 0.335 \\
$\quad$ Cardiac death & $19(1.4)$ & $14(1.6)$ & 0.710 \\
$\quad$ Non-cardiac death & $30(2.2)$ & $11(1.3)$ & 0.103 \\
Any myocardial infarction & $30(2.3)$ & $15(1.8)$ & 0.409 \\
$\quad$ STEMI & $8(0.6)$ & $1(0.1)$ & 0.083 \\
NSTEMI & $22(1.7)$ & $14(1.6)$ & 0.956 \\
Any revascularization & $98(7.4)$ & $62(7.2)$ & 0.892 \\
$\quad$ TLR & $24(1.9)$ & $21(2.5)$ & 0.312 \\
TVR & $48(3.7)$ & $34(4.0)$ & 0.692 \\
NTVR & $51(3.9)$ & $31(3.7)$ & 0.788 \\
Stroke & $27(2.0)$ & $10(1.2)$ & 0.127 \\
\hline
\end{tabular}

MACE major adverse cardiovascular event, STEMI ST-segment elevation myocardial infarction, NSTEMI Non-ST-segment elevation myocardial infarction, NTVR nontarget vessel revascularization, TLR target lesion revascularization, TVR target vessel revascularization

glycemic parameters, while pitavastatin and pravastatin have relatively neutral effects on glycemic control regardless of the presence or absence of DM [1]. Our group recently published a report regarding the favorable glycemic effects of moderate-intensity pitavastatin in comparison to those of moderate-intensity atorvastatin and rosuvastatin in patients with AMI [14]. Despite the current guidelines recommending high-intensity or maximally tolerated statin for secondary prevention in patients with AMI [7, 8], there is no study, to the best of our knowledge, that has compared the diabetogenic effects of different highintensity statins.

Several studies were conducted regarding the cardiovascular outcomes after high-intensity statin therapy. In patients with acute coronary syndrome, both the atorvastatin and rosuvastatin groups had comparable effects

Table 3 Cox regression of clinical outcome

\begin{tabular}{|c|c|c|c|c|}
\hline & \multicolumn{2}{|l|}{ Univariable } & \multicolumn{2}{|l|}{ Multivariable } \\
\hline & Hazard ratio $(95 \% \mathrm{Cl})$ & $p$ value & Hazard ratio $(95 \% \mathrm{Cl})$ & $P$-value \\
\hline \multicolumn{5}{|l|}{ NODM } \\
\hline Age & $0.988(0.976-1.000)$ & 0.052 & & \\
\hline Male gender & $1.307(0.848-2.013)$ & 0.225 & & \\
\hline Glucose & $1.009(1.006-1.011)$ & $<0.001$ & $1.009(1.006-1.011)$ & $<0.001$ \\
\hline Triglyceride & $1.001(1.000-1.002)$ & 0.011 & $1.001(1.000-1.002)$ & 0.021 \\
\hline$\beta$ blocker & $0.768(0.517-1.139)$ & 0.189 & & \\
\hline Statin type & $1.098(0.808-1.491)$ & 0.551 & & \\
\hline \multicolumn{5}{|l|}{ MACE } \\
\hline Age & $1.026(1.016-1.037)$ & $<0.001$ & $1.020(1.009-1.032)$ & 0.001 \\
\hline Male gender & $0.964(0.695-1.336)$ & 0.824 & & \\
\hline LVEF & $0.974(0.961-0.986)$ & $<0.001$ & $0.980(0.966-0.993)$ & 0.003 \\
\hline Glucose & $1.003(1.000-1.006)$ & 0.089 & & \\
\hline Creatinine & $1.155(1.080-1.235)$ & $<0.001$ & $1.204(1.062-1.364)$ & 0.004 \\
\hline LDL cholesterol & $0.995(0.992-0.999)$ & 0.011 & $0.998(0.994-1.002)$ & 0.258 \\
\hline Statin type & $0.944(0.727-1.225)$ & 0.662 & & \\
\hline Ticagrelor or prasugrel & $0.685(0.522-0.899)$ & 0.006 & $0.784(0.585-1.051)$ & 0.104 \\
\hline ACEi or ARB & $0.761(0.569-1.018)$ & 0.066 & & \\
\hline$\beta$ blocker & $0.855(0.609-1.201)$ & 0.367 & & \\
\hline
\end{tabular}


on lipid parameters $[15,16]$, although patients with familial hypercholesterolemia in the rosuvastatin group demonstrated a greater reduction in LDL cholesterol levels than those in the atorvastatin group [17]. Some studies have reported more favorable effects of rosuvastatin on reducing atherosclerotic plaque volume $[15,18]$ and plaque stabilization [15] than of atorvastatin; however, there has been no significant difference with regard to the cardiovascular outcome in both groups $[17,19]$. In line with these previous studies, we could not identify the differences between the effects of high-intensity atorvastatin and rosuvastatin administration on major cardiovascular events.

Several studies have suggested the possible mechanisms underlying the effect of statin on glucose metabolism. Some studies have suggested the interconnection between glucose and lipid metabolisms by demonstrating gene variants affecting glucose metabolism [20-22], cholesterol-dependent conformational change in glucose transporter protein [23], or deleterious effect on islet $\beta$ cells [24] by statins. There is a scarcity of data regarding the mechanisms underlying the different diabetogenic effects of statins; hence, further study would be needed.

This study has several limitations. First, our study is not an RCT, which inevitably leads to selection bias and an imbalance in baseline characteristics. However, as KAMIR is a prospective registry that enrolls Korean patients with AMI, it can represent real-word clinical data. Second, there is a lack of data on rosuvastatin 40 $\mathrm{mg}$ and a relatively small number of patients have been treated with atorvastatin $80 \mathrm{mg}$; hence, dose-dependent increases in NODM could not be demonstrated in our study. Third, this study lacks data regarding the compliance of statin during the follow-up period. Most conventional variables such as age, LVEF, renal function, and new antiplatelet agents were shown to be significant prognostic factors for cardiovascular outcomes, but RAS blockers and $\beta$ blockers were reported as modest and insignificant prognostic factors, respectively. Despite its limitations, to the best of our knowledge, this is the first study from a multicenter registry that demonstrated detailed real-world data on the effect of high-intensity statin on incidence of NODM and MACE in patients with AMI.

\section{Conclusions}

In conclusion, high-intensity atorvastatin therapy showed similar incidence of NODM and cardiovascular events when compared with high-intensity rosuvastatin therapy in patients with AMI. Although prospective, randomized trials with a larger study population are needed to clarify our results, the outcomes presented here provide supportive evidence for the diabetogenic and cardioprotective effects of high-intensity statins in patients with AMI.

\section{Supplementary Information}

The online version contains supplementary material available at https://doi. org/10.1186/s40360-021-00476-z.

\section{Additional file 1.}

\section{Abbreviations}

AMI: acute myocardial infarction; KAMIR: Korea Acute Myocardial Infarction Registry; LDL: low-density lipoprotein; MACE: major adverse cardiac events; NODM: new-onset diabetes mellitus; $\mathrm{PCl}$ : percutaneous coronary

intervention; RCT: randomized controlled trial

\section{Acknowledgements}

This study was done with the support of Korean Circulation Society (KCS). The KAMIR study group of the KSC was as follows: Woong Chol Kang, Gachon University Gil Medical Center, Incheon; Kiyuk Chang, Catholic University Hospital, Seoul; Seung Ho Hur, Keimyung University Dongsan Medical Center, Daegu; Seung Woon Rha, Korea University Guro Hospital, Seoul; Kwang-Soo Cha, Pusan National University Hospital, Busan; Hyeon Cheol Gwon, Samsung Medical Center, Seoul; Seok Kyu Oh, Wonkwang University Hospital, Iksan; Jei Keon Chae, Chonbuk National University Hospital, Jeonju; Kyung-Kook Hwang, Chungbuk National University Hospital, Chungju; Chong Jin Kim, East West Neo Medical Center, Seoul; Jung Han Yoon, Wonju Severance Christian Hospital, Wonju; Jin Yong Hwang, Gyeongsang National University Hospital, Jinju; Doo II Kim, Inje University Busan Paik Hospital, Busan; Seung Jae Joo, Jeju National University Hospital, Jeju. We would like to thank Editage (www.editage.co.kr) for English language editing.

\section{Authors' contributions}

JYC: conception and design of the work, acquisition/analysis/interpretation of data, writing- Original draft preparation, BGC: acquisition/analysis/ interpretation of data, YP, DOK, WYJ, and WK: acquisition/analysis of data, JON, JWK, EJK, SWR, CGP and HSS: acquisition of data, MHJ, SCC, IWS, CHY, KSC, SKO: conception of the work, acquisition of data, CUC: conception and design of the work, supervision, reviewing and editing, acquisition/analysis of data. The author(s) read and approved the final manuscript.

\section{Funding}

This research was supported by a fund (2016-ER6304-02) by Research of Korea Centers for Disease Control and Prevention.

\section{Availability of data and materials}

The datasets generated and/or analysed during the current study are not publicly available because it is confidential, but are available from professor Myung Ho Jeong on reasonable request.

\section{Ethics approval and consent to participate}

This study was approved by the Institutional Review Board of Korea University (IRB No. 2016GR0740) and the Institutional Review Board of each participating hospital. All participants provided written informed consent. Permission to analyze the data was also granted by KAMIR-NIH registry executive.

\section{Consent for publication}

Not applicable.

\section{Competing interests}

The authors declare that they have no competing interests.

\section{Author details \\ ${ }^{1}$ Cardiovascular Center, Korea University Guro Hospital, 80, Guro-dong, Guro-gu, Seoul 152-703, Republic of Korea. ${ }^{2}$ Cardiovascular Center, Catholic University of Korea St. Vincent Hospital, Suwon, Republic of Korea. ${ }^{3}$ Chonnam National University Hospital, Gwangju, Republic of Korea. ${ }^{4}$ Kyungbook National University Hospital, Kyungbook National University School of Medicine, Daegu, Republic of Korea. ${ }^{5}$ Chungnam National University Hospital, Daejeon, Republic of Korea. ${ }^{6}$ Seoul National University Bundang Hospital, Seongnam, South Korea. ${ }^{7}$ Pusan National University Hospital, Busan, South Korea. ${ }^{8}$ Wonkwang University School of Medicine, Iksan, Korea.}


Received: 16 June 2020 Accepted: 25 January 2021

Published online: 04 February 2021

\section{References}

1. Betteridge DJ, Carmena R. The diabetogenic action of statins - mechanisms and clinical implications. Nat Rev Endocrinol. 2016;12(2):99-110.

2. Lee J, Noh Y, Shin S, Lim HS, Park RW, Bae SK, Oh E, Kim GJ, Kim JH, Lee S. Impact of statins on risk of new onset diabetes mellitus: a population-based cohort study using the Korean National Health Insurance claims database. Ther Clin Risk Manag. 2016;12:1533-43.

3. Sattar N, Preiss D, Murray HM, Welsh P, Buckley BM, de Craen AJM, Seshasai SRK, MCMurray JJ, Freeman DJ, Jukema JW, et al. Statins and risk of incident diabetes: a collaborative meta-analysis of randomised statin trials. Lancet. 2010;375(9716):735-42.

4. Preiss D, Seshasai SR, Welsh P, Murphy SA, Ho JE, Waters DD, DeMicco DA, Barter P, Cannon CP, Sabatine MS, et al. Risk of incident diabetes with intensive-dose compared with moderate-dose statin therapy: a metaanalysis. JAMA. 2011;305(24):2556-64.

5. Hiro T, Kimura T, Morimoto T, Miyauchi K, Nakagawa Y, Yamagishi M, Ozaki Y, Kimura K, Saito S, Yamaguchi T, et al. Effect of intensive statin therapy on regression of coronary atherosclerosis in patients with acute coronary syndrome: a multicenter randomized trial evaluated by volumetric intravascular ultrasound using pitavastatin versus atorvastatin (JAPAN-ACS [JAPAN assessment of pitavastatin and atorvastatin in acute coronary syndrome] study). J Am Coll Cardiol. 2009;54(4):293-302.

6. Cannon CP, Steinberg BA, Murphy SA, Mega JL, Braunwald E. Meta-analysis of cardiovascular outcomes trials comparing intensive versus moderate statin therapy. J Am Coll Cardiol. 2006;48(3):438-45.

7. Mach F, Baigent C, Catapano AL, Koskinas KC, Casula M, Badimon L, Chapman MJ, De Backer GG, Delgado V, Ference BA, et al. 2019 ESC/EAS guidelines for the management of dyslipidaemias: lipid modification to reduce cardiovascular risk. Eur Heart J. 2020;41(1):111-88.

8. Grundy SM, Stone NJ, Bailey AL, Beam C, Birtcher KK, Blumenthal RS, Braun LT, de Ferranti S, Faiella-Tommasino J, Forman DE, et al. 2018 AHA/ACC/ AACVPR/AAPA/ABC/ACPM/ADA/AGS/APhA/ASPC/NLA/PCNA guideline on the Management of Blood Cholesterol: a report of the American College of Cardiology/American Heart Association task force on clinical practice guidelines. J Am Coll Cardiol. 2019;73(24):e285-350.

9. Rajpathak SN, Kumbhani DJ, Crandall J, Barzilai N, Alderman M, Ridker PM. Statin therapy and risk of developing type 2 diabetes: a meta-analysis. Diabetes Care. 2009;32(10):1924-9.

10. Ridker PM, Pradhan A, MacFadyen JG, Libby P, Glynn RJ. Cardiovascular benefits and diabetes risks of statin therapy in primary prevention: an analysis from the JUPITER trial. Lancet. 2012;380(9841):565-71.

11. Chen CW, Chen TC, Huang KY, Chou P, Chen PF, Lee CC. Differential impact of statin on new-onset diabetes in different age groups: a population-based case-control study in women from an asian country. Plos One. 2013:8(8):e71817.

12. Carter AA, Gomes T, Camacho X, Juurlink DN, Shah BR, Mamdani MM. Risk of incident diabetes among patients treated with statins: population based study. BMJ. 2013;346:f2610.

13. Maruyama T, Takada M, Nishibori Y, Fujita K, Miki K, Masuda S, Horimatsu T, Hasuike T. Comparison of preventive effect on cardiovascular events with different statins. Circ J. 2011;75(8):1951-9.

14. Choi JY, Choi CU, Hwang SY, Choi BG, Jang WY, Kim DY, Kim W, Park EJ, Lee $\mathrm{S}, \mathrm{Na}$ JO, et al. Effect of Pitavastatin compared with atorvastatin andRosuvastatin on new-onset diabetes mellitus in PatientsWith acute myocardial infarction. Am J Cardiol. 2018;122(6):922-8.

15. Thondapu V, Kurihara O, Yonetsu T, Russo M, Kim HO, Lee H, Soeda T, Minami $Y$, Jang IK. Comparison of Rosuvastatin versus atorvastatin for coronary plaque stabilization. Am J Cardiol. 2019;123(10):1565-71.

16. Altunkeser BB, Tuncez A, Ozturk B, Tezcan H, Ates MS, Yilmaz C, Yalcin MU, Aygul N, Demir K. Comparative effects of high-dose atorvastatin versus rosuvastatin on lipid parameters, oxidized low-density lipoprotein, and proprotein convertase subtilisin kexin 9 in acute coronary syndrome. Coron Artery Dis. 2019;30(4):285-90.

17. Perez de Isla L, Arroyo-Olivares R, Muniz-Grijalvo O, Diaz-Diaz JL, Zambon D, Fuentes F, Sanchez Munoz-Torrero JF, Mediavilla JD, Gonzalez-Estrada A, Miramontes-Gonzalez JP, et al. Long-term effect of 2 intensive statin regimens on treatment and incidence of cardiovascular events in familial hypercholesterolemia: The SAFEHEART study. J Clin Lipidol. 2019;13(6):989-96.
18. Kumar A, Shariff M, Doshi R. Impact of rosuvastatin versus atorvastatin on coronary atherosclerotic plaque volume - a systematic review and metaanalysis with trial sequential analysis of randomized control trials. Eur J Prev Cardiol. 2020:27(19):2138-41.

19. Perez-Calahorra S, Laclaustra M, Marco-Benedi V, Pinto X, SanchezHernandez RM, Plana N, Ortega E, Fuentes F, Civeira F. Comparative efficacy between atorvastatin and rosuvastatin in the prevention of cardiovascular disease recurrence. Lipids Health Dis. 2019;18(1):216.

20. Schmidt AF, Swerdlow DI, Holmes MV, Patel RS, Fairhurst-Hunter Z, Lyall DM, Hartwig FP, Horta BL, Hyppönen E, Power C, et al. PCSK9 genetic variants and risk of type 2 diabetes: a mendelian randomisation study. Lancet Diab Endocrinol. 2017;5(2):97-105.

21. Lotta LA, Sharp SJ, Burgess S, Perry JRB, Stewart ID, Willems SM, Luan J, Ardanaz E, Arriola L, Balkau B, et al. Association between low-density lipoprotein cholesterol-lowering genetic variants and risk of type 2 diabetes: a meta-analysis. JAMA. 2016;316(13):1383-91.

22. Ference BA, Robinson JG, Brook RD, Catapano AL, Chapman MJ, Neff DR, Voros S, Giugliano RP, Davey Smith G, Fazio S, et al. Variation in PCSK9 and HMGCR and risk of cardiovascular disease and diabetes. N Engl J Med. 2016; 375(22):2144-53.

23. Nowis D, Malenda A, Furs K, Oleszczak B, Sadowski R, Chlebowska J, Firczuk M, Bujnicki JM, Staruch AD, Zagozdzon R, et al. Statins impair glucose uptake in human cells. BMJ Open Diabetes Res Care. 2014;2(1):e000017.

24. Sampson UK, Linton MF, Fazio S. Are statins diabetogenic? Curr Opin Cardiol. 2011;26(4):342-7.

\section{Publisher's Note}

Springer Nature remains neutral with regard to jurisdictional claims in published maps and institutional affiliations.
Ready to submit your research? Choose BMC and benefit from:
- fast, convenient online submission
- thorough peer review by experienced researchers in your field
- rapid publication on acceptance
- support for research data, including large and complex data types
- gold Open Access which fosters wider collaboration and increased citations
- maximum visibility for your research: over $100 \mathrm{M}$ website views per year
At BMC, research is always in progress.
Learn more biomedcentral.com/submissions 